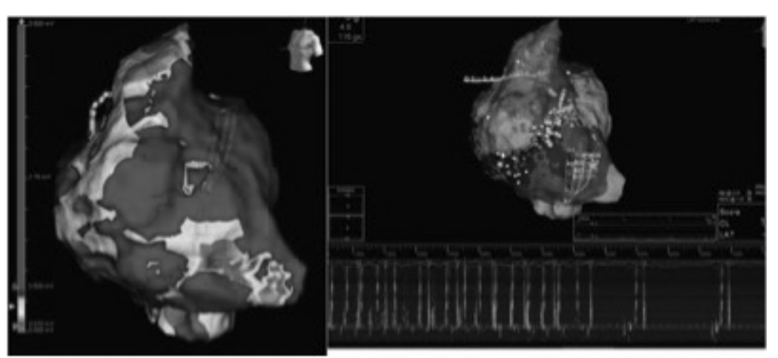

Abstract 137 Figure 1 Voltage map (L) and re-entrant map (R) of an AT in an AP Fontan patient made using a 16-pole-4 spline grid catheter using HD wave solutions. Re-entry is seen around an area of functional block, and ablation from the line of block (black) to inferolateral scar resulted in termination to SR at superior end of line ( $R$ panel elctrograms).

Across all cases, 472,072 points were collected in an average time of 15 min with HD and 92,026 with conventional bipoles. The mean procedure and fluoroscopy times were $180 \pm 69$ and $7 \pm 5$ min respectively. $58 \%$ cases were performed without using any fluoroscopy. There were no procedural complications.

with HD and 92,026 with conventional bipoles. Within the critical isthmus, the mean voltage amplitude and number of points using $\mathrm{HD}$ wave was higher than conventional bipoles $(1.21 \mathrm{mV}, 92$ versus $0.86 \mathrm{mV}, 76)$. Critical isthmus sites were missed in 4 micro re-entrant ATs cases using conventional bipoles alone. Ablation at the identified critical isthmus led to arrhythmia termination in all cases. There were no procedural complications. Arrhythmia free survival was $78 \%$ at a mean follow-up of 6 months.

Conclusion This novel mapping approach was able to accurately define critical isthmus by mapping complex wavefronts using orthogonal bipoles and the "best voltage duplicate" algorithm in complex CHD patients. HD mapping enabled identification of critical channels in areas of scar, which were missed on conventional mapping due to limitations in bipolar density and single orientations meaning wavefront propagation is not fully defined. This is particularly relevant for multiple micro re-entrant ATs in ACHD.

Figure 1: Voltage map (L) and re-entrant map (R) of an AT in an AP Fontan patient made using a 16-pole-4 spline grid catheter using HD wave solutions. Ablation from line of block (black) to inferolateral scar with termination to SR at superior end of line.

Conflict of Interest Nil

\section{NATURAL HISTORY OF ATRIAL FIBRILLATION AND ATRIAL FIBRILLATION ABLATION IN PATIENTS UNDERGOING PERCUTANEOUS ATRIAL SEPTAL DEFECT CLOSURE}

${ }^{1}$ Alexander Carpenter* ${ }^{2}$ Oliver Crowther, ${ }^{2}$ Alexander Gall, ${ }^{2}$ Sarah Elgamal ${ }^{3}$ Richard Bennett, ${ }^{2}$ Mohamed Mehisen, ${ }^{2}$ Mark Turner, ${ }^{3}$ Ashley Nisbet. ${ }^{1}$ Taunton and Somerset NHS Foundation Trust; ${ }^{2}$ Bristol Heart Institute; ${ }^{3} \mathrm{NHS}$

\subsection{6/heartjnl-2019-BCS.135}

Introduction Atrial septal defects (ASD) often co-exist with atrial fibrillation (AF). Current thinking suggests AF burden may be improved by ASD closure and that catheter interventions should occur before closure to minimise procedural risks. There are few published studies investigating the natural history of AF or ablation outcomes in ASD patients.
Methods: We undertook a retrospective observational study of all percutaneous ASD closure procedures over a 12-year period. Demographic and procedural data were collected. Patients with insufficient documentation were excluded. Outcomes at one year follow-up were collected, including death, freedom from AF (office ECG and symptom-led Holter) and stroke/transient ischaemic attack (TIA). Statistical analysis was undertaken using a two-tailed Fisher's exact T-test. Results: From April 2005 to May 2017, 384 percutaneous ASD closures occurred, with 21 excluded from our dataset due to incomplete data. $69 \%$ of patients were female, with a median age of 49 (range 16-84). 88\% of patients had only a single defect. $96 \%$ of procedures were acutely successful, with $3 \%$ repeat procedures. Some residual leak was seen in $10 \% .64 \%$ had follow-up data at one year: all were alive with $1 \%$ incidence of stroke/TIA. $74(20 \%)$ of the cohort had AF prior to closure, in whom $15(20 \%)$ underwent AF ablation (40\% paroxysmal, $60 \%$ persistent). $11(73 \%)$ were ablated prior to closure. Overall, $80 \%$ of the ablation cohort were free from $\mathrm{AF}$ at one year with no strokes/TIA, with $100 \%(\mathrm{~N}=6 / 6)$ success in the paroxysmal group $(67 \%$ in the persistent group $\mathrm{N}=6 / 9 ; \mathrm{p}=0.49$ ). There was no significant difference with AF ablation prior to closure: $82 \%$ AF-free at one year, versus $75 \%$ AF-free in the ablation after closure group, $(\mathrm{p}=1)$. Of those AF patients who did not undergo ablation, $36 \%$ were AF-free at one year following closure. Of 289 patients $(80 \%)$ without any pre-existing $\mathrm{AF}$, rates of progression to AF by one year were remarkably low, with only a single patient receiving a new diagnosis $(0.3 \%)$. Conclusions: Our data demonstrate favourable short and long-term outcomes supporting ASD closure as a safe intervention with a low failure rate. We characterise the prevalence of AF in this cohort including the natural history with and without ablation. AF ablation enjoys 1-year success rates comparable to a non-ASD closure population. There is a signal, though nonstatistically significant, that ablation for paroxysmal AF enjoys increased success rates. Interestingly, we see that following closure, there may be a degree of regression of AF in those even without ablation. We observed very low rates of new AF diagnoses in the follow-up period. Our cohort represents a real-world experience of several hundred consecutive patients undergoing ASD closure over more than a decade, and provides a significant new body of data in a field where many mechanistic and therapeutic questions remain unanswered.

Conflict of Interest Nil

\section{IDENTIFICATION OF THE MAJOR GENETIC CONTRIBUTORS TO TETRALOGY OF FALLOT}

${ }^{1}$ Donna Page* ${ }^{2}$ Simon Williams, ${ }^{2}$ Richard Monaghan, ${ }^{2}$ Elisavet Fotiou, ${ }^{3}$ Bernard Keavney. ${ }^{1}$ Manchester Metropolitan University; ${ }^{2}$ University of Manchester; ${ }^{3}$ Faculty of Biology, Medicine and Health, University of Manchester

\subsection{6/heartjnl-2019-BCS.136}

Introduction There is strong evidence from familial recurrence studies for a genetic predisposition to sporadic, non-syndromic Tetralogy of Fallot (TOF). TOF is the most common, cyanotic congenital heart disease (CHD) phenotype yet the cause for the majority of cases remains elusive. Rare genetic variants have been identified as important contributors to the risk of $\mathrm{CHD}$, but relatively small numbers of TOF cases have been studied to date. 